\title{
Transcranial Electrical Brain Stimulation
}

\section{() (1) $\circledast$}

Authors

Janine Reis, Brita Fritsch

Affiliation

Department of Neurology, Albert- Ludwigs-Universität Freiburg, Germany

Key words

noninvasive brain stimulation, stroke, neuroplasticity, neurorehabilitation

Bibliography

DOI http://dx.doi.org/10.1055/s-0043-102478

Neurology International Open 2017; 1: E142-E147

(c) Georg Thieme Verlag KG Stuttgart · New York

ISSN 2511-1795

Correspondence

Dr. Janine Reis

Department of Neurology

Albert- Ludwigs-Universität Freiburg
Breisacher Straße 64

79106 Freiburg

janine.reis@uniklinik-freiburg.de

\section{ABSTRACT}

Transcranial electrical brain stimulation using weak direct current (tDCS) or alternating current (tACS) is being increasingly used in clinical and experimental settings to improve cognitive and motor functions in healthy subjects as well as neurological patients. This review focuses on the therapeutic value of transcranial direct current stimulation for neurorehabilitation and provides an overview of studies addressing motor and nonmotor symptoms after stroke, disorders of attention and consciousness as well as Parkinson's disease.

\section{Background}

The past 10 years have seen an increased clinical and experimental focus on noninvasive electrical brain stimulation as an innovative therapeutic approach to support neurorehabilitation. This entails the application of either transcranial direct current stimulation (tDCS), or less commonly, transcranial alternating current stimulation (tACS). Typically, up to $0.8 \mathrm{~A} / \mathrm{m}^{2}$ is used for up to $40 \mathrm{~min}$ per single stimulation session [1]. The electrical current partially penetrates the underlying structures and affects nerve cells, glia and vessels in the stimulated brain area [1,2]. Early animal experiments during the 1960s and 1970s on the effects of weak DC stimulation demonstrated an excitement-induced change of neurons lasting several hours after the end of the stimulation $[3,4]$. Therapeutic studies of the 1970 s, at that time mainly concerning the treatment of depression, did not yield any success, which in retrospect could be attributed to the stimulation parameters used. In 2000 key experiments by Nitsche and Paulus on polarity-related excitability changes in the human motor system after transcranial application of tDCS led to a renewed interest in the approach [5]. The authors documented increased cortical excitability measured by the amplitude of motor-evoked potentials in healthy volunteers after anodal stimulation above the motor cortex lasting at least 9 min [6]. Reversing the direction of stimulation (cathodal tDCS) resulted in a decrease in motor-evoked potential. In addition to the concept of pure excitability modulation, a large number of studies demonstrate modulation of neuroplasticity by tDCS in various ways, including basic scientific and mechanistic findings regarding improvement of synaptic transmission strength [7-9], long-term influence on learning processes and behavior $[10,11]$, as well as a therapeutic approach to improve function in neurological and psychiatric disorders associated with altered or disturbed neuroplasticity (overview in [12]). In particular, simultaneous application of tDCS together with different learning paradigms, such as motor or cognitive training, appears to produce favorable effects in healthy subjects and in various patient groups [11, 13].

The following review presents the effects of tDCS on the improvement in the function of some neurological disease patterns which are regularly the focus of neurorehabilitative treatment. This especially includes stroke. In addition, we shall refer to a current database of clinical studies containing a comprehensive list of scientific and clinical studies of tDCS in the treatment of neurological and psychiatric disorders [14].

\section{Post-stroke Motor Impairment}

Stroke is one of the primary causes worldwide of permanent limitations of motor function and speech. Despite intensive rehabilitation efforts, approx. $50 \%$ of stroke patients remain limited in their 


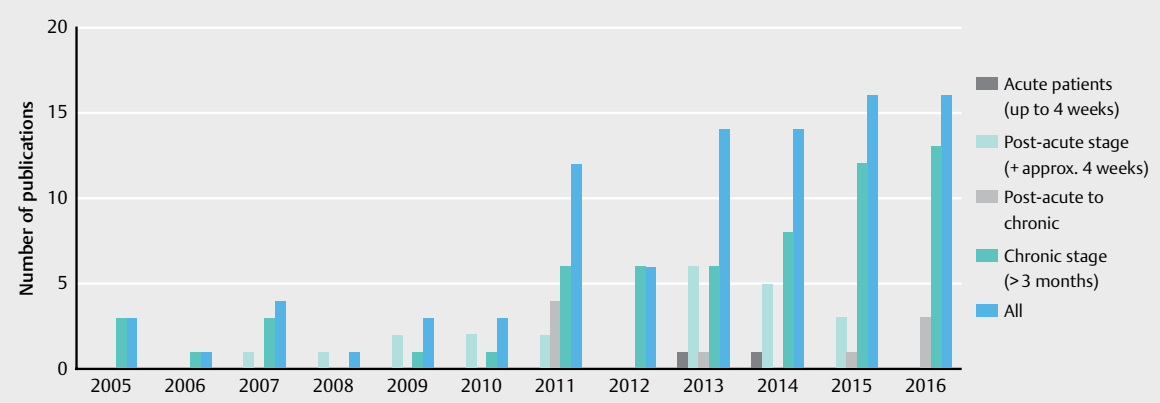

-Fig. 1 Graphical representation of original work published per year on tDCS used in the treatment of stroke patients with motor impairment symptoms. Although the greater number of studies were performed involving patients in the chronic stage ( $>3$ months after episode), there are hardly any describing the acute phase ( $<4$ weeks, dark grey bar). This has to be taken into account when evaluating the value of tDCS for neurorehabilitation.

motor and speech capabilities [15-17]. Current understanding of the mechanisms of tDCS is largely based on data documented for the human motor system. The reasons for this include the presence of direct and easily objectifiable measurement criteria (for example, motor-evoked potential, fine motor function), as well as anatomical accessibility of brain motor regions for non-invasive stimulation. Therefore, it is not surprising that the clinical syndrome of stroke with the frequent symptom of hemiparesis as a "lesion model of the pyramidal tract" received significant scientific interest with respect to researching the effects of tDCS, as evidenced by the numerous scientific publications since 2005 ( $\vee$ Fig. 1). In contrast to earlier largely mechanistic studies, in the past 5 years there has been a trend toward studies addressing clinically-oriented therapeutic issues.

After a stroke, a cortical environment is created by the brain injury itself which promotes neuroplastic processes. Electrophysiological data and results from functional imaging confirm active involvement of both hemispheres (ipsilesional and contralesional) on functional recovery [18-20], with a varying role at different times after an acute event. Based on the overall concept of interhemispheric competition, the data for patients show a poorer recovery if there is persistent functional imbalance between the 2 hemispheres (over-activation of the unaffected hemisphere) [18]. This leads to the general consideration that tDCS can be used to restore the functional balance between the 2 hemispheres, either by activating (anodal) stimulation of the affected hemisphere, or by suppressing (cathodal) stimulation of the unaffected hemisphere ( $\triangleright$ Fig. 2). Both of these stimulation montages involving the motor cortex are used routinely. An additional stimulation protocol has been used since 2010, that is, the simultaneous stimulation of both motor cortices (anode ipsilesionally, cathode contralesionally). It should be kept in mind that tDCS, as a relatively unfocal technique, not only reaches the actual target area (for example the motor cortex), but also stimulates other nearby brain regions [1]. Furthermore, the simplified concept of excitement or suppression of cortical function is only a guideline, and individual factors influencing complex cortical network interactions as well as the passage of time after stroke must also be considered.

The current data on the use of tDCS in the treatment of motor impairment after a stroke are reflected in a number of individual studies describing the promising effects of tDCS on upper limb function, especially when used in combination with therapeutic procedures (physio-/occupational therapy, CIMT, robot-supported training, fine-motor training) applied in repeated sessions $[12,21,22]$. The improvement of motor skills of the upper limb in comparison to sham stimulation ranges between 5 and $30 \%$, depending on the study and outcome measure applied (e. g., Fugl Meyer score, Wolf motor function test, ARAT, grip force, specific kinematic parameters). In addition to the heterogeneity of the outcome measure, the type of intervention (motor training, physio-/ occupational therapy, robot-assisted therapy) and the stimulation itself (time, intensity, duration and montage/target area for tDCS) also vary in the studies. With few exceptions, the patient cohorts are small and heterogeneous. Although early proof-of-principle studies are not included, a current Cochrane meta-analysis concludes that due to the study heterogeneity to date point and given the low quality of the available studies, there is little evidence that tDCS provides an improvement in daily activities, although there is evidence for a lack of improvement in motor deficits [23]. If only the motor function of the upper extremities is specifically evaluated as a primary outcome measure, other meta-analyses show a possible positive effect of tDCS in combination with rehabilitation programs [24]. When only specific motor training is considered as an intervention, there is a clearly positive effect of tDCS on upper extremity motor function compared to sham stimulation or rehabilitation interventions alone [25]. It should be mentioned that the inclusion and exclusion criteria as well as the primary endpoints differ in all meta-analyses.

In summary, at this time it can be assumed that tDCS has a positive effect on the improvement of daily activities as well as motor functions after a stroke. However, in order to support this assumption, 2 essential aspects should be addressed in the future: 1 . the time window post-stroke, 2 . study heterogeneity. The meta-analyses named above are based on data which for the most part were gathered from patients in the chronic stage (3 months to years) after suffering a stroke. Thus there is a lack of studies on the effects of tDCS on patients in the acute phase post-stroke. As shown in - Fig. 1, to date only 2 studies of acute patients have been published [26, 27]. In both studies tDCS was applied without simultaneous therapy or training, and was ineffective compared to sham 
a

Activation of damaged hemisphere

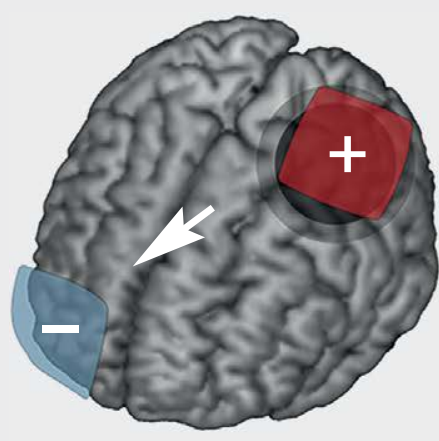

b
Suppression of undamaged hemisphere c

Simultaneous activation of damaged hemisphere/suppression of undamaged hemisphere

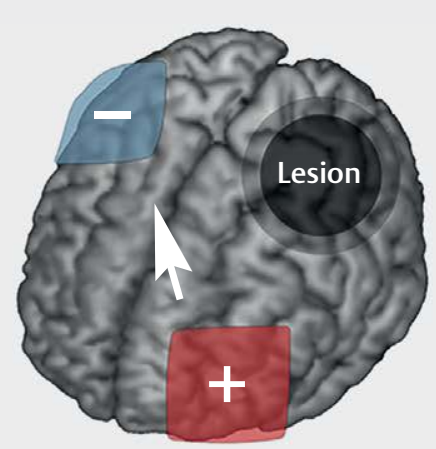

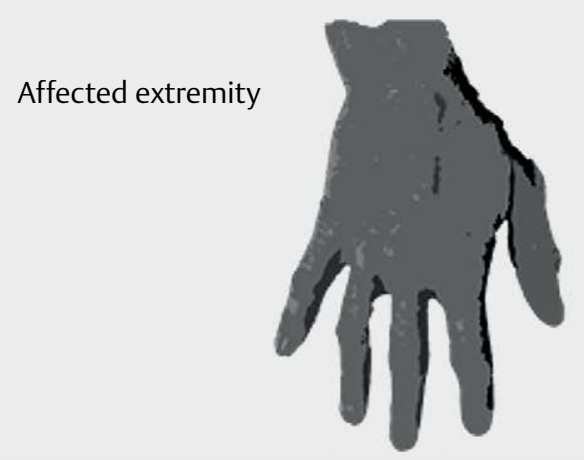

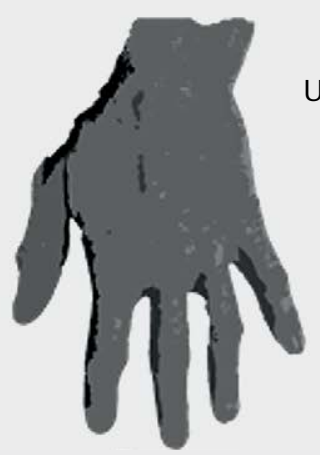

Unaffected extremity

-Fig. 2 Illustration of the 3 typical brain stimulation montages exemplified by tDCS above the motor cortex. In example a, the anode (red) is placed above the ipsilesional motor cortex, and the cathode (blue) is located on the contralateral forehead. Example b shows the cathode placed above the motor cortex of the non-lesioned hemisphere, and the anode is placed on the contralateral forehead. Example $\mathrm{c}$ illustrates bihemispheric montage, with the anode located above the ipsilesional motor cortex, and the cathode placed above the motor cortex of the non-lesioned hemisphere. The white arrow shows the intracerebral current flow. The goal of these 3 arrangements is to modulate the interaction between both motor cortices by changing the activity of one or both hemispheres $\mathbf{c}$.

stimulation. Due to the plastic environment present in the acute phase after stroke, the targeted combination of tDCS and therapy could have promising effects on the rehabilitation process or even extend the "plastic phase". Current studies are focusing specifically on this aspect, including our own research work (Emmy Noether program "Mechanisms and Modulation of Motor Skill Learning in the healthy and injured Brain", as well as a large-scale multi-center study (DFG, NETS TRIAL: Neuroregeneration enhanced by tDCS in stroke, Study Coordinator: C. Gerloff, Hamburg). Further controlled, randomized, prospective multi-center clinical trials are needed in order to substantiate the value of tDCS and timing in the rehabilitation of motor functions after stroke. In addition, evaluation of interaction of tDCS and disease-associated neuroplastic changes must be supported by basic scientific research. To date 5 experimental animal studies on tDCS after acute stroke have been published [28-32]. A common feature of all these studies is repeated tDCS under anesthesia. Interaction between both modalities can not be ruled out, since both act on the excitability of neurons. A combination of tDCS with training or rehabilitation has not yet been tested; this is equivalent to the isolated assessment of modulation of spontaneous biological recovery. The current densities used are well above the human application of $0.8 \mathrm{~A} / \mathrm{m}^{2}$, even above the lesion threshold for anodal tDCS in a healthy rat [2]. With these parameters there was a tendency towards neurodegenerative effects for anodal stimulation and somewhat positive effects for cathodal stimulation with respect to behavior and infarct volume [3032]. In the 2 studies with the lowest current density ( 1 and $28 \mathrm{~A}$ / $\mathrm{m}^{2}$, both transcutaneous) anodal tDCS had a positive effect on the rat's cognitive and motor behavior without affecting the infarct volume [28, 29]. In order to successfully translate research into the clinical context and the mechanistic understanding of the diseased brain (interaction of pathology and stimulation effect), further basic research studies are also necessary, the design of which should include application in a conscious animal as well as stimulation parameters mimicking the human conditions.

\section{Aphasia and Neglect after Stroke}

Disturbances of non-motor functions and spatial perception are also common after a stroke. Analogously to the hemisphere concept with respect to motor impairment, there is a similar rationale for the application of tDCS in cases of hemispatial neglect or apha- 
sia: targeted modulation of the affected brain region or surrounding perilesional areas on the one hand and modulation of the intact hemisphere on the other. Available literature relating to neglect is currently limited. Unlike studies of motor impairment, to date there are only proof of concept studies in which tDCS is mainly used alone, i. e., without simultaneous perceptual training and applied only once. Repeated sessions have been investigated in only 3 studies [33-35]; in the first, feedback training was performed, and in the second, a combination using prism adaptation was used as training. Compared to sham stimulation, the proof of concept studies as well as studies with repeated application demonstrate a reduction of neglect symptoms in specific tests (line bisection, find and cross-out tasks) after anodal stimulation of the right, i. e., ipsilesional posterior parietal cortex or cathodal stimulation of the left, unaffected posterior parietal cortex. Interestingly, synchronous bihemispheric tDCS of both posterior parietal cortices has a variable outcome in studies [34,35], which might indicate an intraindividually different role of both hemispheres in the functional recovery from neglect. There are currently no meta-analyses.

For those affected by a stroke, speech impairment is frequently a debilitating restriction on communication and interaction with the world around them. Therefore there is great clinical interest in evaluating tDCS as adjuvant therapy in the treatment of speech impairment after stroke, as demonstrated by the number of publications on the topic (see the database in [14]). The effects of tDCS combined with speech therapy or conversation training were evaluated, and with few exceptions, the results demonstrate overall positive effects among patients in the chronic stage, although variable effects have been described for the subacute stage [36-38]. The target area for stimulation in case of aphasia is clearly more heterogeneous (see [37]); individual studies have reported positive effects of anodal tDCS for the frontal inferior gyrus and posterior gyrus temporalis superior of the damaged hemisphere. Similarly positive results have been reported for cathodal tDCS above the frontal inferior gyrus of the undamaged hemisphere. Interestingly, stimulation of the left motor cortex, which anatomically lies further remote from the structural lesion of the primary language region improved the ability to communicate. This could be explained by increased interaction with the individual's residual language network [39]. The primary outcome measures were mainly naming ability, word fluidity and word recognition. A meta-analysis with naming ability as the primary outcome measure showed a significant advantage of tDCS compared to control conditions in patients in the chronic stage post-stroke [40]. A further meta-analysis, in which the primary target criterion "communicative ability" could not be assessed due to a lack of data , the secondary outcome measure "naming ability"; outcome measure revealed no significant effect of tDCS combined with speech therapy compared to sham stimulation and speech therapy calculated over 6 studies with similar mean difference [41]. In the latter meta-analysis no distinction was made between different phases post-stroke.

\section{Consciousness and Attentiveness}

Impairment of attention and wakefulness, such as after a traumatic brain injury, can have a significant influence of the outcome of rehabilitation. Application of transcranial brain stimulation in this regard has been explored only in minor case reports and open clinical studies. In patients in a minimally conscious state [42], anodal tDCS above the left dorsolateral prefrontal cortex achieved short-lasting improvements of clinically-measurable reactions, brain stem reflexes and arousal, whereas patients with apallic syndrome had no benefit [43]. Mixed effects of tDCS applied over the DLPFC could be shown for single and repeated stimulation prior to cognitive training in patients with traumatic brain injury and chronic persistent memory and attention deficits. Whereas Lesniak and colleagues [44] found no effect of tDCS, positive effects of tDCS prior to cognitive training with regard to specific subdomains such as divided attention, word interference and impulse control have been reported $[45,46]$.

\section{Chronic Motor and Non-motor Symptoms}

\section{of Parkinson's Disease}

In addition to the manifold studies of stroke in which tDCS is used to intervene functionally with the sensimotor and attention system, similar questions arise with respect to neurodegenerative diseases involving motor and non-motor symptoms such as Parkinson's disease. To date there are few studies in this regard. Investigations of patients with idiopathic Parkinson's syndrome reveal a favorable effect of tDCS on specific symptoms, such as improvement of working memory after stimulation of the dorsolateral prefrontal cortex $[47,48]$, or reduction of dyskinesia after anodal stimulation of the motor cortex or cerebellum [49]. There are recent scientific approaches to the treatment of disorders of the cortex-basal ganglia axis such as applying alternating current stimulation (tACS) or alternating current stimulation with frequencies varying randomly between 100 and $640 \mathrm{~Hz}$ (transcranial random noise stimulation, tRNS). The purpose is to influence intrinsic cortical and subcortical oscillations (in the sense of entrainment or phase cancellation [50,51]). Initial results for tremor-dominant Parkinson's syndrome show a $50 \%$ reduction of resting tremor amplitude under tACS [50]. The only 2 basic studies of tDCS in a Parkinson's rat model show positive effects of anodal stimulation on behavioral parameters (partial normalization of side preference [52], longer running times in the rotarod test [53]). From a mechanistic point of view, the dopamine and tyrosine hydroxylase concentrations in the entire brain were increased, and the concentration of oxidative stress parameters was reduced compared to sham stimulation, suggesting potentially neuroprotective effects of anodal tDCS. Combinations with rehabilitation or training have yet to be investigated. Further multimodal studies on the potential neuroprotective effects of tDCS in a dose-response design or at least comparable to stimulation parameters applied to humans are important for useful clinical application.

\section{Safety Considerations}

There are a large number of studies available allowing the estimation of the safety of tDCS. To date more than 1000 volunteers and patients in more than 33200 sessions using tDCS have been examined and treated (using conventional stimulation protocols discussed above) with no reports of serious adverse events [1]. Typi- 
cal side effects of all non-invasive electrical brain stimulation procedures include itching and burning sensations on the scalp, headache, reddening of the skin in the area under the electrodes and general feelings of discomfort; however such sensations have also been reported for sham stimulation $[1,54]$. Currently there are no reports regarding triggering of epileptic episodes, and data based on animal experiments using epilepsy models indicate that tDCS itself poses no increased risk of seizures in an epileptic brain [1]. It should be noted that physiological and behavioral effects of tDCS in traumatic brain injury, especially in the presence of skull defects, may be more variable, since changes in the intracranial current flow direction are possible and thus brain regions other than expected are affected. The same considerations apply to cerebral lesions. The latest computational models calculate an up to 6-fold higher intracerebral current density in patients with skull defects; however this does not represent a general safety risk and is therefore not an exclusion criterion for the presently used stimulation parameters (up to $0.8 \mathrm{~A} / \mathrm{m}^{2}$ ) [1].

\section{Summary}

Positive effects of tDCS have been reported for a number of neurological symptoms that are in the focus of neurorehabilitation, especially with respect to long-term improvement of daily competencies, reduction of neglect in the subacute phase as well as aphasia reduction in the chronic phase after stroke. In the future, studies of acute patients as well as basic mechanistic research are needed to support informed clinical application of tDCS in neurorehabilitation.

\section{Conflict of Interest}

No conflict of interest has been declared by the authors.

\section{References}

[1] Bikson M, Grossman P, Thomas C et al. Safety of transcranial direct current stimulation: Evidence based update 2016. Brain Stimul 2016; 9: 641-661

[2] Gellner A-K, Reis ], Fritsch B. Glia: A neglected player in non-invasive direct current brain stimulation. Front Cell Neurosci 2016; 10: 188

[3] Bindman LJ, Lippold OC, Redfearn JW. Long-lasting changes in the level of the electrical activity of the cerebral cortex produced bypolarizing currents. Nature 1962; 196: 584-585

[4] Gartside IB. Mechanisms of sustained increases of firing rate of neurones in the rat cerebral cortex after polarization: role of protein synthesis. Nature 1968; 220: 382-383

[5] Nitsche M, Paulus W. Excitability changes induced in the human motor cortex by weak transcranial direct current stimulation. J Physiol 2000; 527 (Pt 3): 633-639

[6] Nitsche MA, Paulus W. Sustained excitability elevations induced by transcranial DC motor cortex stimulation in humans. Neurology 2001; 57: 1899-1901

[7] Fritsch B, Reis J, Martinowich K et al. Direct current stimulation promotes BDNF-dependent synaptic plasticity: Potential implications for motor learning. Neuron 2010; 66: 198-204
[8] Podda MV, Cocco S, Mastrodonato A et al. Anodal transcranial direct current stimulation boosts synaptic plasticity and memory in mice via epigenetic regulation of Bdnf expression. Sci Rep 2016; 6: 22180

[9] Ranieri F, Podda MV, Riccardi E et al. Modulation of LTP at rat hippocampal CA3-CA1 synapses by direct current stimulation. J Neurophysiol 2012; 107: 1868-1880

[10] Reis ], Schambra HM, Cohen LG et al. Noninvasive cortical stimulation enhances motor skill acquisition over multiple days through an effect on consolidation. Proc Natl Acad Sci U S A 2009; 106: 1590-1595

[11] Buch ER, Santarnecchi E, Antal A et al. Effects of tDCS on motor learning and memory formation: A consensus and critical position paper. bioRxiv 2016 Available at http://biorxiv.org/content/early/2016/07/18/064204.abstract

[12] Lefaucheur J-P, Antal A, Ayache SS et al. Evidence-based guidelines on the therapeutic use of transcranial direct current stimulation (tDCS). Clin Neurophysiol 2016; 128: 56-92

[13] Cappon D, Jahanshahi M, Bisiacchi P. Value and efficacy of transcranial direct current stimulation in the cognitive rehabilitation: A critical review since 2000. Front Neurosci 2016; 10: 157

[14] Lefaucheur JP. A comprehensive database of published tDCS clinical trials (2005-2016). Clin Neurophysiol 2016; 46: 319-398

[15] Verheyden G, Nieuwboer A, Wit LD et al. Time course of trunk, arm, leg, and functional recovery after ischemic stroke. Neurorehabil Neural Repair 2007 Available at http://www.ncbi.nlm.nih.gov/entrez/query. fcgi?cmd = Retrieve\&db = PubMed\&dopt = Citation\&list_ uids $=17876069$

[16] Kwakkel G, Kollen B, Twisk J. Impact of time on improvement of outcome after stroke. Stroke 2006; 37: 2348-2353

[17] Flowers HL, Skoretz SA, Silver FL et al. Poststroke aphasia frequency, recovery, and outcomes: A systematic review and meta-analysis. Arch Phys Med Rehabil 2016; 97: 2188-2201.e8

[18] Grefkes C, Ward NS. Cortical reorganization after stroke: How much and how functional? Neuroscientist 2014; 20: 56-70

[19] Duque J, Hummel F, Celnik P et al. Transcallosal inhibition in chronic subcortical stroke. Neuroimage 2005; 28: 940-946

[20] Murase N, Duque ], Mazzocchio R et al. Influence of interhemispheric interactions on motor function in chronic stroke. Ann Neurol 2004; 55: 400-409

[21] Allman C, Amadi U, Winkler AM et al. Ipsilesional anodal tDCS enhances the functional benefits of rehabilitation in patients after stroke. Sci Transl Med 2016; 8: 330re1

[22] Mortensen J, Figlewski K, Andersen H. Combined transcranial direct current stimulation and home-based occupational therapy for upper limb motor impairment following intracerebral hemorrhage: A double-blind randomized controlled trial. Disabil Rehabil 2016; 38 : 637-643

[23] Elsner B, Kugler ], Pohl M et al. Transcranial direct current stimulation (tDCS) for improving activities of daily living, and physical and cognitive functioning, in people after stroke. Cochrane database Syst Rev 2016; 3: CD009645

[24] Tedesco Triccas L, Burridge JH, Hughes AM et al. Multiple sessions of transcranial direct current stimulation and upper extremity rehabilitation in stroke: A review and meta-analysis. Clin Neurophysiol 2016; 127: 946-955

[25] Kang N, Summers J], Cauraugh JH. Transcranial direct current stimulation facilitates motor learning post-stroke: A systematic review and meta-analysis. J Neurol Neurosurg Psychiatry 2016; 87: 345-355

[26] Rossi C, Sallustio F, Di Legge S et al. Transcranial direct current stimulation of the affected hemisphere does not accelerate recovery of acute stroke patients. Eur J Neurol 2013; 20: 202-204 
[27] Di Lazzaro V, Dileone M, Capone F et al. Immediate and late modulation of interhemipheric imbalance with bilateral transcranial direct current stimulation in acute stroke. Brain Stimul 2014; 7: 841-848

[28] Kim S], Kim BK, Ko Y] et al. Functional and histologic changes after repeated transcranial direct current stimulation in rat stroke model. J Korean Med Sci 2010; 25: 1499-1505

[29] Yoon K], Oh B-M, Kim D-Y. Functional improvement and neuroplastic effects of anodal transcranial direct current stimulation (tDCS) delivered 1 day vs. 1 week after cerebral ischemia in rats. Brain Res 2012; 1452: 61-72

[30] Peruzzotti-Jametti L, Cambiaghi M, Bacigaluppi M et al. Safety and efficacy of transcranial direct current stimulation in acute experimental ischemic stroke. Stroke 2013; 44: 3166-3174

[31] Notturno F, Pace M, Zappasodi F et al. Neuroprotective effect of cathodal transcranial direct current stimulation in a rat stroke model. J Neurol Sci 2014; 342: 146-151

[32] Braun R, Klein R, Walter HL et al. Transcranial direct current stimulation accelerates recovery of function, induces neurogenesis and recruits oligodendrocyte precursors in a rat model of stroke. Exp Neurol 2016; 279: 127-136

[33] Yi YG, Chun MH, Do KH et al. The effect of transcranial direct current stimulation on neglect syndrome in stroke patients. Ann Rehabil Med 2016; 40: 223-229

[34] Làdavas E, Giulietti S, Avenanti A et al. a-tDCS on the ipsilesional parietal cortex boosts the effects of prism adaptation treatment in neglect. Restor Neurol Neurosci 2015; 33: 647-662

[35] Bang D-H, Bong S-Y. Effect of combination of transcranial direct current stimulation and feedback training on visuospatial neglect in patients with subacute stroke: a pilot randomized controlled trial. J Phys Ther Sci 2015; 27: 2759-2761

[36] Torres J, Drebing D, Hamilton R. TMS and tDCS in post-stroke aphasia: Integrating novel treatment approaches with mechanisms of plasticity. Restor Neurol Neurosci 2013; 31: 501-515

[37] Monti A, Ferrucci R, Fumagalli M et al. Transcranial direct current stimulation (tDCS) and language. J Neurol Neurosurg Psychiatry 2013; 84: 832-842

[38] Hamilton RH, Chrysikou EG, Coslett B. Mechanisms of aphasia recovery after stroke and the role of noninvasive brain stimulation. Brain Lang 2011; 118: 40-50

[39] Meinzer M, Darkow R, Lindenberg R et al. Electrical stimulation of the motor cortex enhances treatment outcome in post-stroke aphasia. Brain 2016; 139: 1152-1163

[40] Shah-Basak PP, Wurzman R, Purcell JB et al. Fields or flows? A comparative metaanalysis of transcranial magnetic and direct current stimulation to treat post-stroke aphasia. Restor Neurol Neurosci 2016; 34: $537-558$
[41] Elsner B, Kugler J, Pohl M et al. Transcranial direct current stimulation (tDCS) for improving aphasia in patients with aphasia after stroke. Cochrane database Syst Rev 2015; CD009760

[42] Giacino JT, Ashwal S, Childs $\mathrm{N}$ et al. The minimally conscious state: definition and diagnostic criteria. Neurology 2002; 58: 349-353

[43] Thibaut A, Bruno M-A, Ledoux D et al. tDCS in patients with disorders of consciousness: Sham-controlled randomized double-blind study. Neurology 2014; 82: 1112-1118

[44] Leśniak M, Polanowska K, Seniów J et al. Effects of repeated anodal tDCS coupled with cognitive training for patients with severe traumatic brain injury: A pilot randomized controlled trial. J Head Trauma Rehabil 2014; 29: E20-E29

[45] Ulam F, Shelton C, Richards L et al. Cumulative effects of transcranial direct current stimulation on EEG oscillations and attention/working memory during subacute neurorehabilitation of traumatic brain injury. Clin Neurophysiol 2015; 126: 486-496

[46] Sacco K, Galetto V, Dimitri D et al. Concomitant use of transcranial direct current stimulation and computer-assisted training for the rehabilitation of attention in traumatic brain injured patients: behavioral and neuroimaging results. Front Behav Neurosci 2016; 10: 57

[47] Doruk D, Gray Z, Bravo GL et al. Effects of tDCS on executive function in Parkinson's disease. Neurosci Lett 2014; 582: 27-31

[48] Manenti R, Brambilla M, Benussi A et al. Mild cognitive impairment in Parkinson's disease is improved by transcranial direct current stimulation combined with physical therapy. Mov Disord 2016; 31: 715-724

[49] Ferrucci R, Cortese F, Bianchi M et al. Cerebellar and motor cortical transcranial stimulation decrease levodopa-induced dyskinesias in parkinson's disease. Cerebellum 2016; 15: 43-47

[50] Brittain J-S, Probert-Smith P, Aziz TZ et al. Tremor suppression by rhythmic transcranial current stimulation. Curr Biol 2013; 23: 436-440

[51] Vosskuhl J, Strüber D, Herrmann CS. Transcranial alternating current stimulation. Entrainment and function control of neuronal networks. Nervenarzt 2015; 86: 1516-1522

[52] Li Y, Tian X, Qian L et al. Anodal transcranial direct current stimulation relieves the unilateral bias of a rat model of Parkinson's disease. Conf Proc IEEE Eng Med Biol Soc 2011; 2011: 765-768

[53] Lu C, Wei Y, Hu R et al. Transcranial direct current stimulation ameliorates behavioral deficits and reduces oxidative stress in 1-methyl-4-phenyl-1,2,3,6-tetrahydropyridine-induced mouse model of parkinson's disease. Neuromodulation 2015; 18: 442-446 discussion 447

[54] Brunoni AR, Amadera J, Berbel B et al. A systematic review on reporting and assessment of adverse effects associated with transcranial direct current stimulation. Int ] Neuropsychopharmacol 2011; $14: 1133-1145$ 